\title{
Design of Higher Order Modulation Schemes for Orthogonal Frequency Division Multiplexing (OFDM) Systems
}

\author{
P. Naga Malleswari \\ CVR College of Engineering /ECE, Hyderabad, India \\ Email: pinjalamalleswari@gmail.com.
}

\begin{abstract}
In this paper we design and simulate OFDM (Orthogonal Frequency Division Multiplexing) to study the performance of the OFDM system at higher order modulation schemes. It is very important to evaluate performance of the communication system, to test the efficiency and quality of the service it can provide. We use Matlab program to design the functionality of OFDM, then BER(Bit Error Rate) is obtained to different SNR(Signal to Noise Ratio) values, for M-array PSK and M-array QAM modulations techniques. BER is widely used as performance measurement tool, it tells number of bits destroyed while the data is travelling from source to the destination, AWGN(Additive White Gaussian Noise) is used as transmission channel.
\end{abstract}

Index Terms-OFDM, Bit error rate, QAM, noise, AWGN.

\section{I.INTRODUCTION}

Orthogonal Frequency Division Multiplexing (OFDM) uses multiple sub-carriers for data transmission. In this transmission scheme all the sub-carriers are orthogonal to each other. OFDM is the combination of multi carrier modulation and multiplexing i.e. it is the process of mapping digital data on multiple carrier frequencies sharing bandwidth with other independent channels. In this modulation technique data symbols modulate the orthogonally separated sub-carriers. This technique is similar to FDM technique except that the $\mathrm{N}$ nonoverlapping Sub-carrier signals are made orthogonal. Unlike other conventional frequency multiplexing techniques, it overcomes the problem of bandwidth wastage by using overlapping but orthogonal sub-carriers.

In this paper the higher order digital modulation techniques that are used for Orthogonal Frequency Division Multiplexing (OFDM) designed. In OFDM we set frequency constant to maintain orthogonality of the sub-carriers and alter amplitude or phase or both at same time. We also discuss communication channels and fading channels, which are used to calculate Bit Error Rate (BER) and the system performance of OFDM transmission technology. In the modulation process to convey the message properly the base band signal is imposed on to high frequency carrier signal and is transmitted without disturbing the original data for long distances. Further we are going to discuss about different modulation techniques and their effect on carrier signal. Frequency of the carrier signal is always higher than the frequency of base band signal. In digital modulation the modulating signal is digital bit stream. Digital modulation is classified into different types based on the variation in the characteristics of carrier signal, Three characteristics of the signal that can be varied over time are amplitude, phase and frequency. After modulation any one of the three parameters is changed and the other two remains constant. The three fundamental techniques of digital modulation are, Amplitude shift keying (ASK), Frequency shift keying (FSK) and Phase shift keying (PSK).

\section{OFDM Signal generation}

The block diagram of orthogonal Frequency Division Multiplexing is shown in figure 1 .

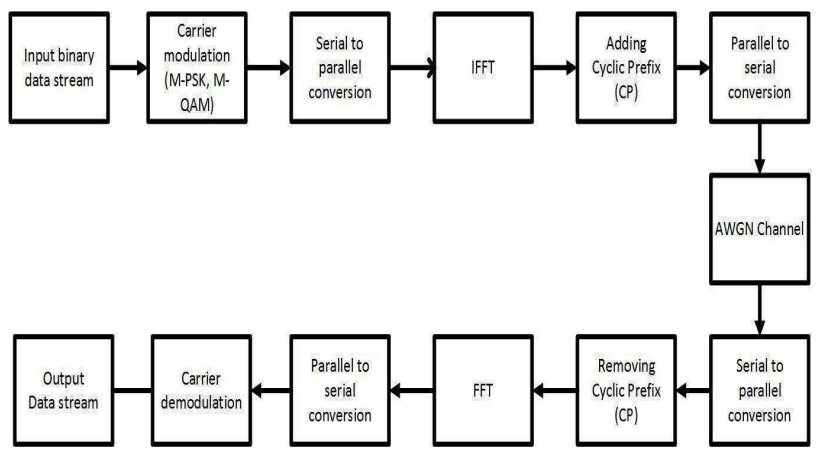

Figure 1: block diagram of OFDM.

\section{A. Transmitter}

Here we discuss the steps involved in transmitting the OFDM signal. Initially the information to be conveyed is given as input to the system as binary data. Unlike other modulation techniques using a single carrier for modulation, OFDM system uses a number of subcarriers one for each symbol of input bit stream. To accomplish this input serial stream of data is converted to parallel streams. Consider a serial bit stream of 3 bits and it is converted in to parallel, Now, each stream is mapped with a complex symbol stream using PSK/QAM. The reason behind choosing particularly these techniques is, they are high level modulation techniques. Mapping using BPSK, QPSK or QAM helps to increase the data rate of OFDM. In this paper the BER vs. SNR of all the three modulations techniques observed. Simple 
illustration of the mechanism going on in this block is given below,

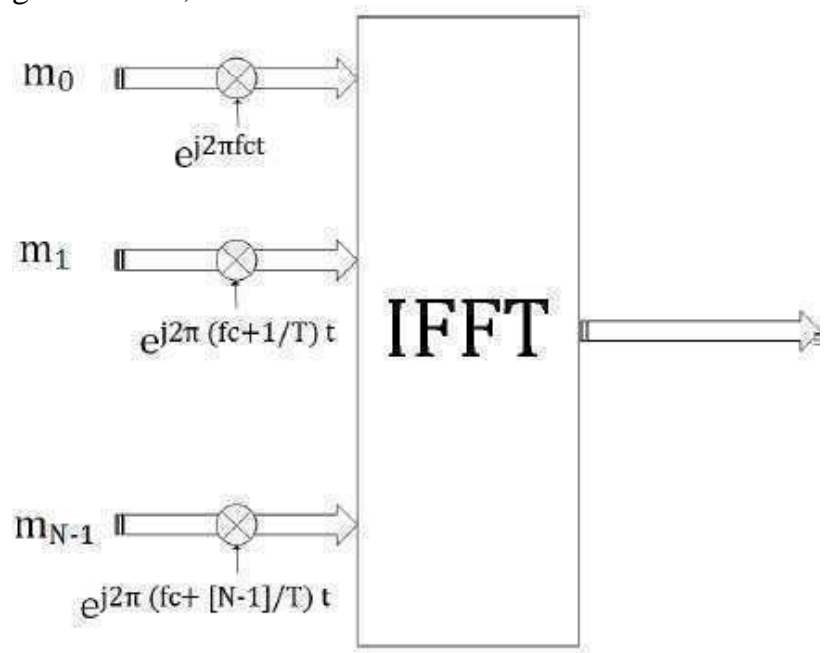

Figure 2: Figure showing conceptual modulation block

In the figure $2 . \mathrm{m}_{0}, \mathrm{~m}_{1}, \ldots . . \mathrm{m}_{\mathrm{N}-1}$ represents $\mathrm{N}$ parallel bit streams obtained after serial to parallel conversion and each branch corresponds to a sub-carrier. Each subcarrier modulates a symbol $\mathrm{m}_{\mathrm{k}}$. To maintain orthogonality, frequency spacing of $1 / \mathrm{T} \mathrm{Hz}$ is maintained between the successive sub-carriers. This is because sinusoidal signals differing in the frequency $1 / \mathrm{T}$ will be orthogonal over the period $\mathrm{T}$.

$$
\int_{t_{n}}^{t_{n}+T} e^{j 2 \pi f_{c} t}\left[e^{-j 2 \pi\left(f_{c}+\frac{1}{T}\right) t}\right] d t=0
$$

Individual sub-carriers are arranged, such that the frequency separation between two successive sub-carriers is $\Delta \mathrm{f}=1 / \mathrm{T}$. Complex symbol streams obtained from modulation block are set as input to the Inverse Fast Fourier Transform (IFFT) block is shown in figure 3. where the domain changes takes place. Usually data obtained after mapping parallel data streams onto subcarriers is in frequency domain. IFFT converts the data in frequency domain function to time domain function. Obtained time domain functions are given as input to parallel to serial converter and the signal are multiplexed. The final output from IFFT is multiplexed time domain signal.

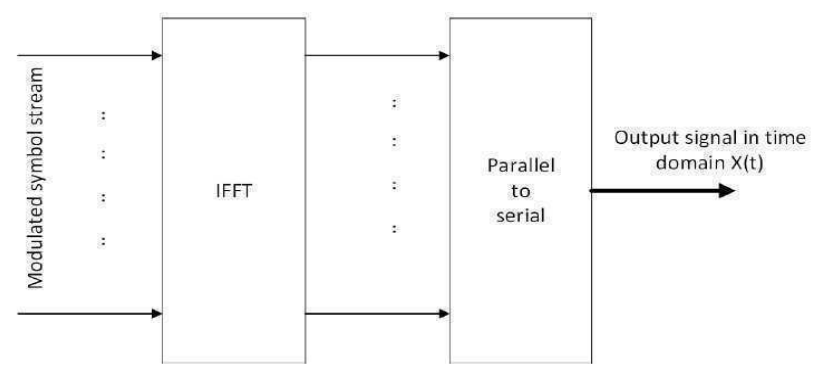

Figure 3: IFFT block diagram
If $\mathrm{N}$ modulated symbol streams are set as input to IFFT with a symbol duration $\mathrm{T}$, then the output OFDM symbol duration is NT. The output signal $\mathrm{X}(\mathrm{t})$ in time domain acts as base band signal for OFDM system. Addition of cyclic prefix To this OFDM signal cyclic prefix is appended to avoid power loss due to echoes. It is generated by prefixing a symbol with its last samples. Important point to be considered in order to serve effectively is its length. Length of the cyclic prefix should be at least equal to the delay of its multipath channel. Apart from this it retains sinusoids properties.

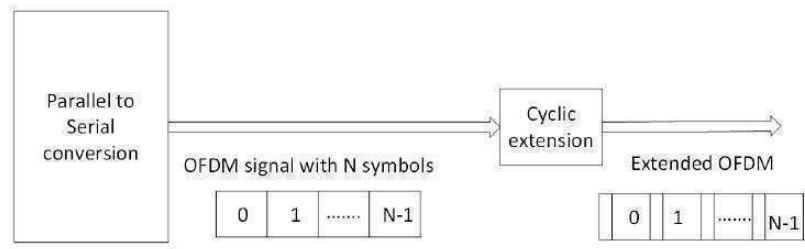

Figure 4: shows the multiplexed OFDM signal with $\mathrm{N}$ symbols.

This is given as input to cyclic prefix extension where cyclic prefix is appended to each symbol. It also shows the difference between OFDM and its extended version. In normal signal which is obtained after multiplexing, all the $\mathrm{N}$ symbols are together one after the other but in case of extended OFDM signal there are samples appended to each symbol shown in figure 5. Thereby the two successive symbols do not interfere with each other. Now the question may arise, which samples are added as prefix and to which extent? What is the use of appending? These questions are answered in the further sections of this chapter. Clear view of extended version of OFDM symbol answers half of our questions.

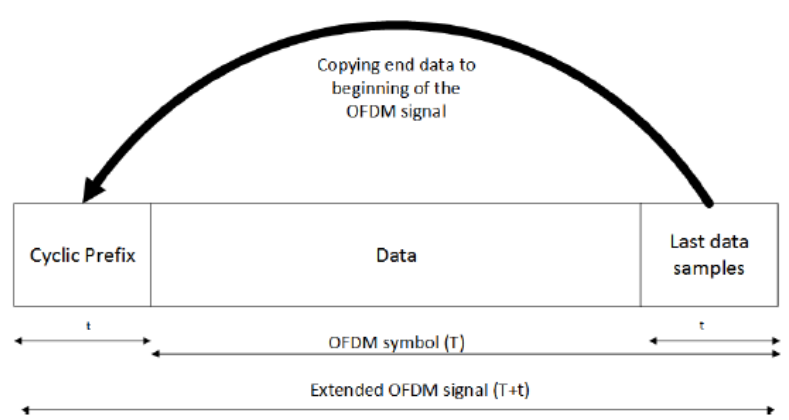

Figure 5: OFDM symbol with cyclic prefix.

The Figure 5 shows the OFDM signal with and without cyclic prefix. It is clearly shown that last samples of the symbol are added as prefix. In the figure last samples and the prefix are in same color and length (4), which indicates that both the samples are same. The length of prefix depends on the delay of multi path channel. It should be longer than the excess delay of longest significant echo. Length of cyclic prefix (4) is also called as guard interval. This extended version of OFDM signal is transmitted through a channel to the receiver. 
B. Effects of Inter Symbol Interference (ISI) and Inter Carrier Interference (ICI) on OFDM

The two interferences ISI, ICI are usually originated by transmission channel. When these interferences are not introduced, orthogonality between the sub-carriers can be maintained and the individual subchannels can be completely separated at the receiver. Practically it is easily achieved. This is because OFDM is not strictly band limited, due to this linear distortions such as multipath makes each sub channel to spread its energy to adjacent channels. This leads to Inter Carrier Interference. Simple solution to prevent this is to increase symbol duration.ISI can be avoided by taking care of guard interval while gluing it to the OFDM symbol, which should be longer than the excess delay of multipath channel.

\section{Communication channels}

In this paper The Additive White Gaussian Noise (AWGN) channel used. This is because we analyze different modulation techniques and AWGN is considered as universal channel for analyzing different modulation schemes. In this, the channel doesn't introduce any distorts expect the addition of white Gaussian noise to the signal passing through it. There by the channels amplitude response is at and its phase frequency response is linear for all frequencies. So Modulated signals pass through it without any amplitude loss or phase distortion. Apart from this fading doesn't exist. There by the received signal is the summation of original signal and white Gaussian noise.

\section{RECEIVER}

First and foremost step at the receiver is removal of appended cyclic prefix, which is equivalent to removal of guard interval.

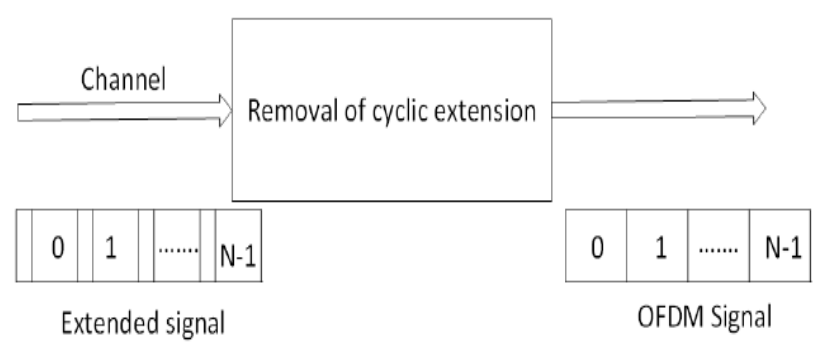

Figure 6: Removal of cyclic extension

After removal of extension the signal is converted back to normal OFDM signal, followed by serial to parallel conversion. While the effect of channel transforms into periodic convolution of discrete time channel with IFFT of data symbols. Performing FFT on received samples converts the periodic convolution to multiplication.

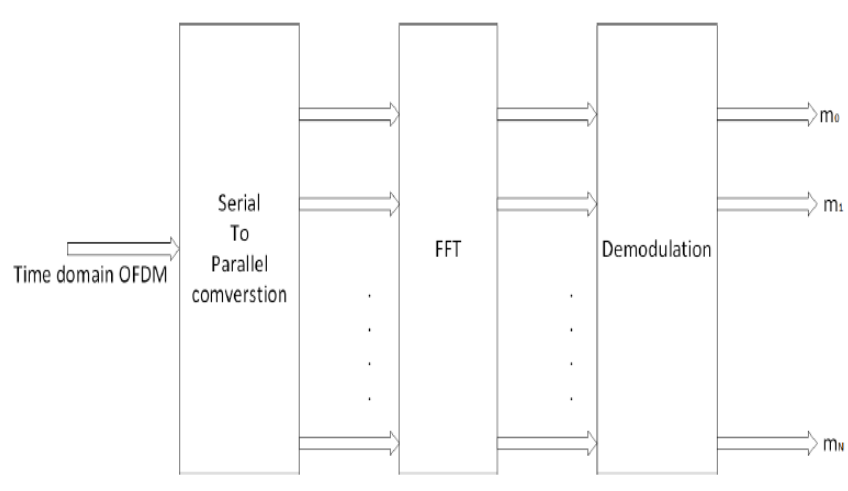

Figure 7:FFT Block diagram

Figure 7. clearly shows the input and output of FFT. FFT gets parallel steams of data in time domain as input, it converts time OFDM signal to frequency domain. Output from FFT is set as input to PSK or QAM demodulator. Demodulator separates bit streams from the carrier and gives parallel bit steams $\mathrm{m}_{0}, \mathrm{~m}_{1} \ldots . \mathrm{m}_{\mathrm{N}-1}$ as output. These bit steams are multiplexed using parallel to serial converter as shown in figure 8 . and the final outcome the message signal to be conveyed. Output from serial to parallel converter is $\mathrm{S}[\mathrm{n}]$, which is given as input to OFDM system.

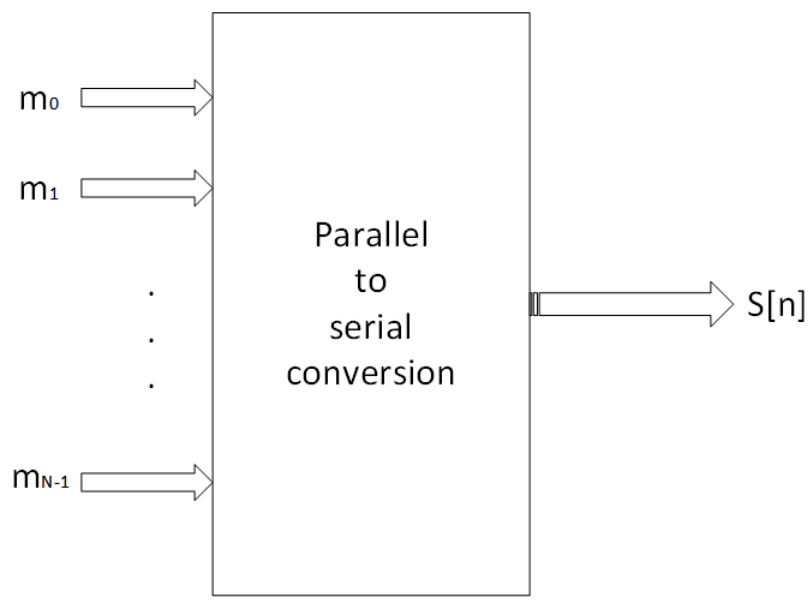

Figure 8: Parallel to serial conversion

\section{IMPLEMENTATION AND RESULTS}

In this chapter we investigate performance of OFDM, for different PSK modulations in AWGN channels. MATLAB program is used to obtain results and calculated SER (Symbol error rate) for various SNR (Signal to Noise Ration). We should note here that BER (Bit Error Rate) can be obtained by,

symbolrate $=\frac{\text { Bitrate }}{\text { Numberofbitstransmittedforsymbol }}$ 


\section{A. PSK modulation scheme}

In M-array PSK modulation system, I-component and Q-component are interdependent, with constant envelop which makes the data points to form in circular constellation [7]. The important goals in designing a digital communication system is to have very low error probability and conservation of bandwidth. In this section we use QPSK,16-PSK and 32-PSK modulations, with AWGN channel. The performance of the system is examined with different SNR values. The results obtained is then plotted and analysed.

\section{B. QPSK modulation}

QPSK uses only half the channel bandwidth used by binary PSK, has same error probability as of binary PSK system with same bit rate and same $\mathrm{E}_{\mathrm{b}} / \mathrm{No}$ [7]. In this Matlab program OFDM system is provided with 64000 binary data, $\mathrm{M}$ modulation level is set to 4 , FFT length is set as 64, cyclic prefix is set to 10 . The Table 1.shows the QPSK Simulation parameters in the OFDM.

TABLE I.

QPSK SIMULATION PARAMETERS.

\begin{tabular}{|c|r|}
\hline \multicolumn{2}{|c|}{ QPSK } \\
\hline M & 4 \\
Input binary data & 64000 \\
No. of symbols & 32000 \\
FFT length & 64 \\
Cyclic Prefix & 10 \\
No. of frames & 500 \\
Frame size & 64 \\
\hline
\end{tabular}

The constellation points of QPSK is shows in figure 9. For QPSK (Quadrature Phase shift Keying) we know it has four constellation points with two I values and two Q values.

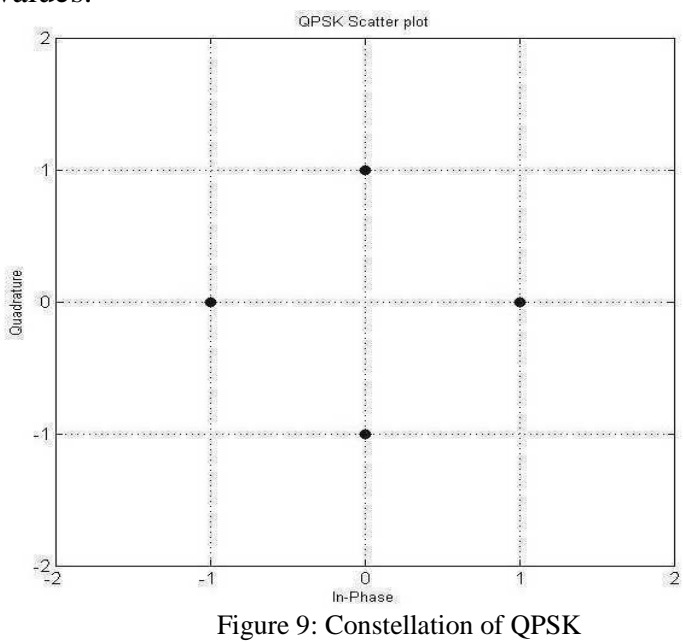

Plots in figure 10. shows the impulsive response and frequency response of the channel and Figure 11. show first 100 input and 100 output samples. The plot in figure 12 , is used to visualize the error data, the red line shown in the graph is the error bit.
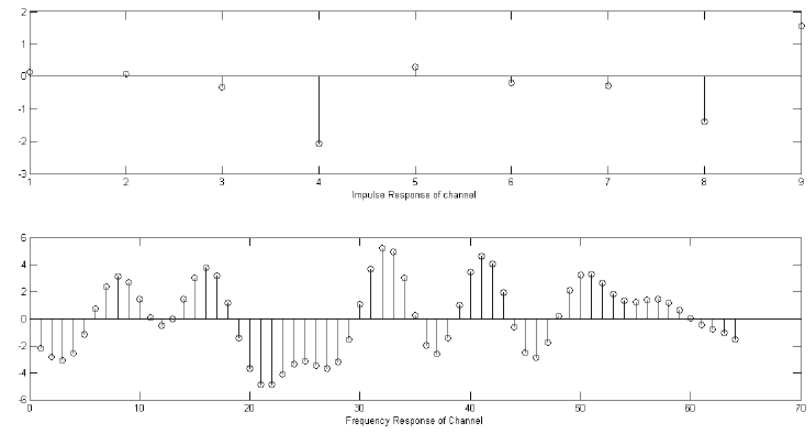

Figure 10: Impulsive and Frequency response of channel
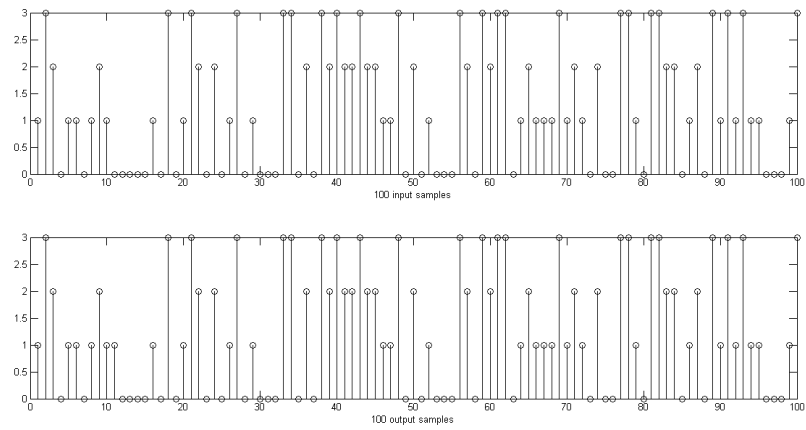

Figure 11: Input and Output samples.

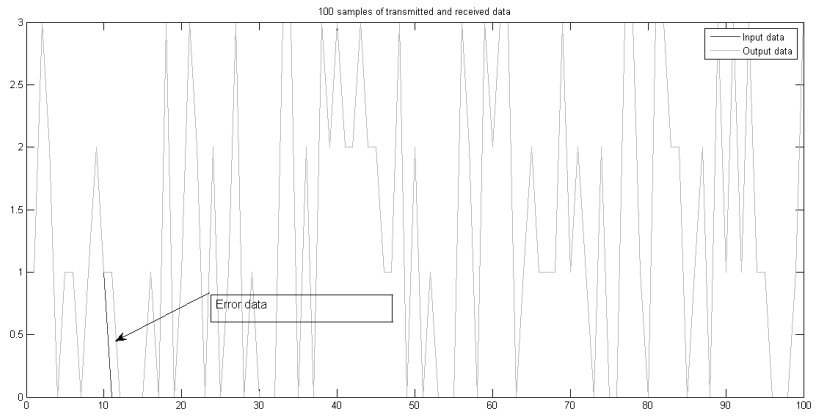

Figure 12: Plot of Input and Output signal to visualize errors.

Figure 13 shows number of errors to respective Signal to noise ratio. In figure 14 is plot of Symbol error rate Vs Signal to noise ratio for QPSK-OFDM system. Apart from this it provides same data rate as BPSK for half the bandwidth needed by BPSK. But the main problem in using QPSK is the complexity of transmitters and receivers. 


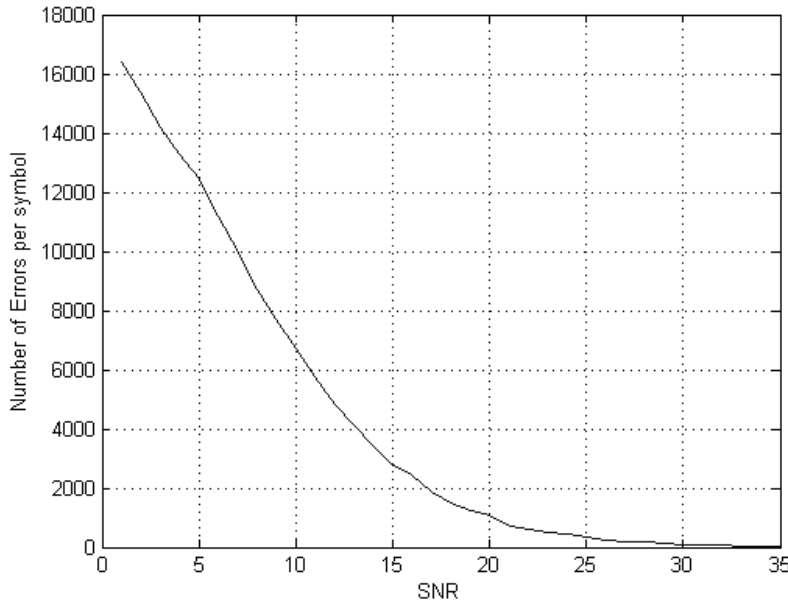

Figure 13: Number of error Vs Signal to noise ratio for QPSK-OFDM.

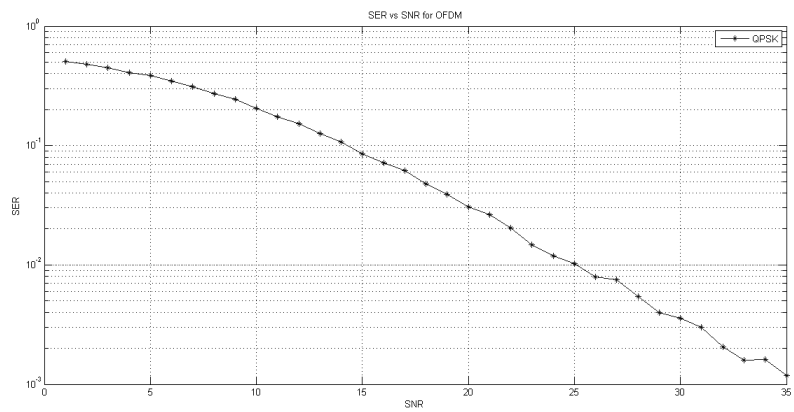

Figure 14: Symbol error rate Vs Signal to noise ratio for QPSK-OFDM.

\section{16PSK modulation}

In 16PSK system we can have 4 bits/symbol. Modulation level $\mathrm{M}$ is set to 16 , total number of bits to be transmitted is 64000 , FFT length is set as 64 and cyclic prefix is 10 .

TABLE II 16PSK SIMULATION PARAMETERS

\begin{tabular}{|r|r|}
\hline \multicolumn{1}{|c|}{ QPSK } \\
\hline M & 16 \\
Input binary data & 64000 \\
No. of symbols & 16000 \\
FFT length & 64 \\
Cyclic Prefix & 10 \\
No. of frames & 250 \\
Frame size & 64 \\
\hline
\end{tabular}

Figure 15. shows 16 points in a circle with phase_22:50 and the demodulator has only _ 11:25o phase to detect the symbol.

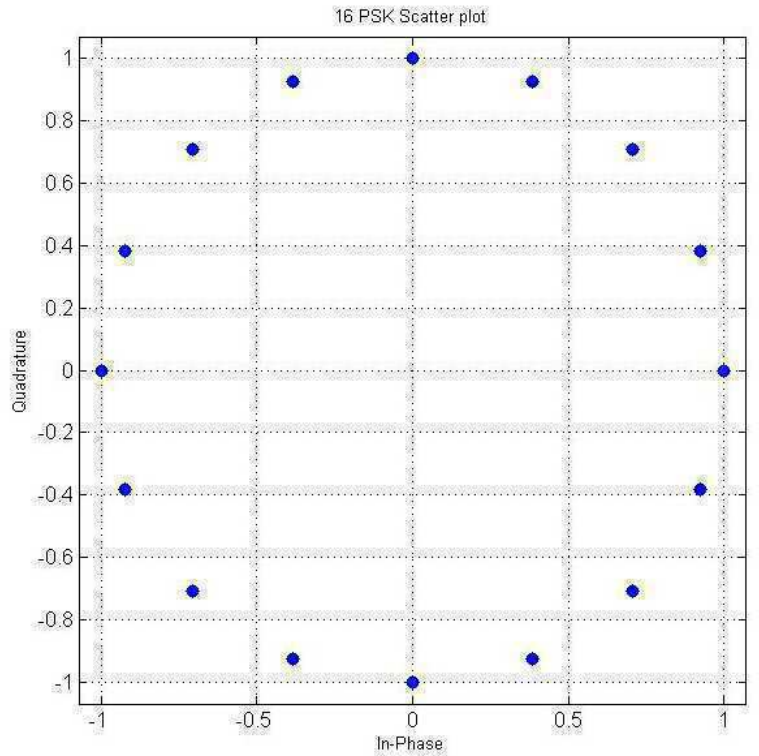

Figure 15: Constellation of 16PSK

Figure 16. show the graphs of first 100 input and output data, the red lines shows where errors has occurred.

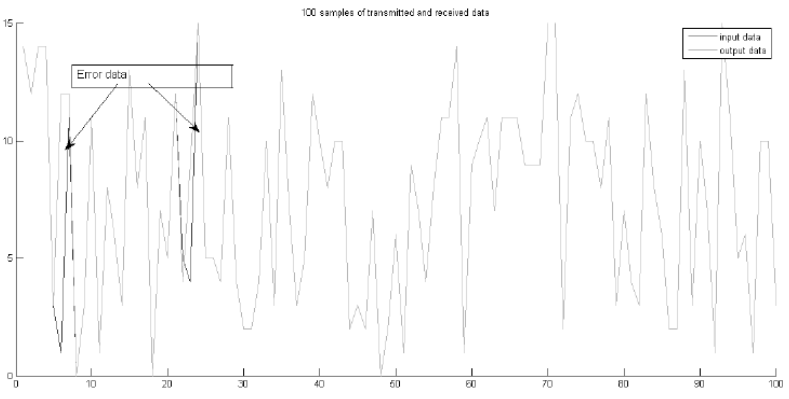

Figure 16: Plot of Input and Output signal to visualize errors.

Figure 17. shows number of errors to respective Signal to noise ratio.

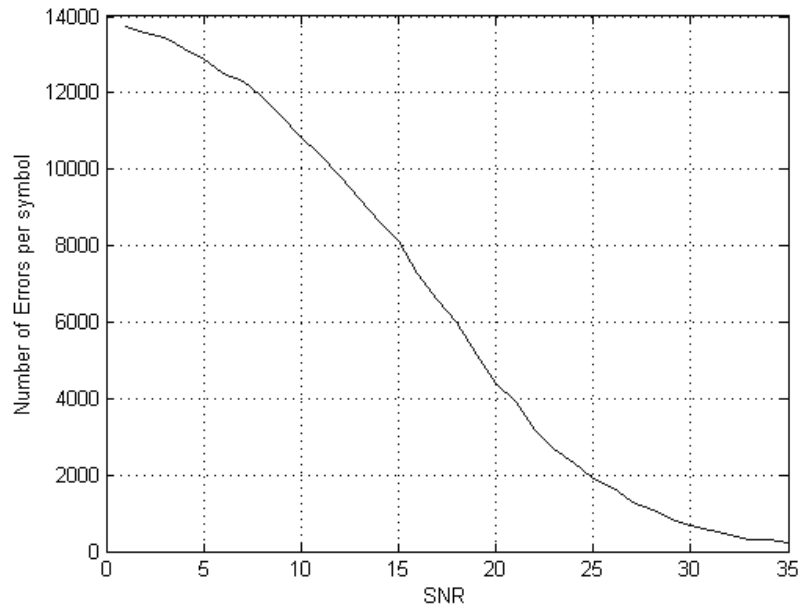

Figure 17: Number of error Vs Signal to noise ratio for 16psk-ofdm

Plot in figure 18. shows SER of the system with different SNR values. 


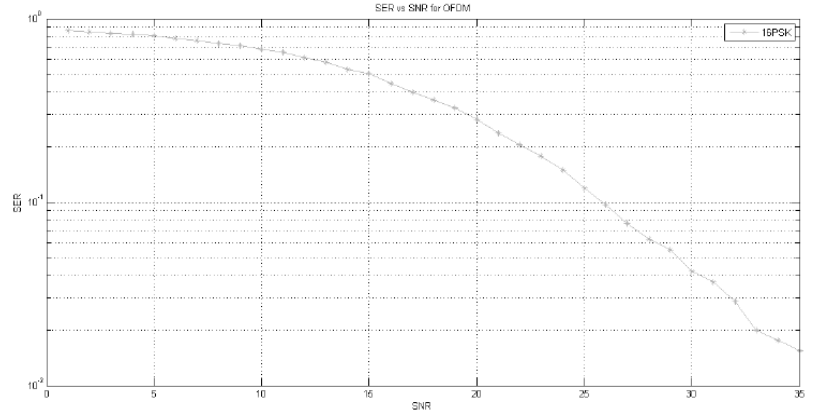

Figure 18: Symbol error rate Vs Signal to noise ratio for 16psk-ofdm

\section{D.32PSK modulation}

In 32-PSK we have 5 bits/symbol, Modulation level is set as 32, total number of bits to transmit is 64000 and FFT length is 64 and cyclic prefix is set as 10 . The constellation diagram shown in figure 19 has 32 points separated with phase of _11:25o and the demodulator has _5:625o phase to detect the symbols.

TABLE III.

THE 32PSK SIMULATION PARAMETERS

\begin{tabular}{|r|r|}
\hline \multicolumn{2}{|c|}{ QPSK } \\
\hline M & 32 \\
Input binary data & 64000 \\
No. of symbols & 12800 \\
FFT length & 64 \\
Cyclic Prefix & 10 \\
No. of frames & 200 \\
Frame size & 64 \\
\hline
\end{tabular}

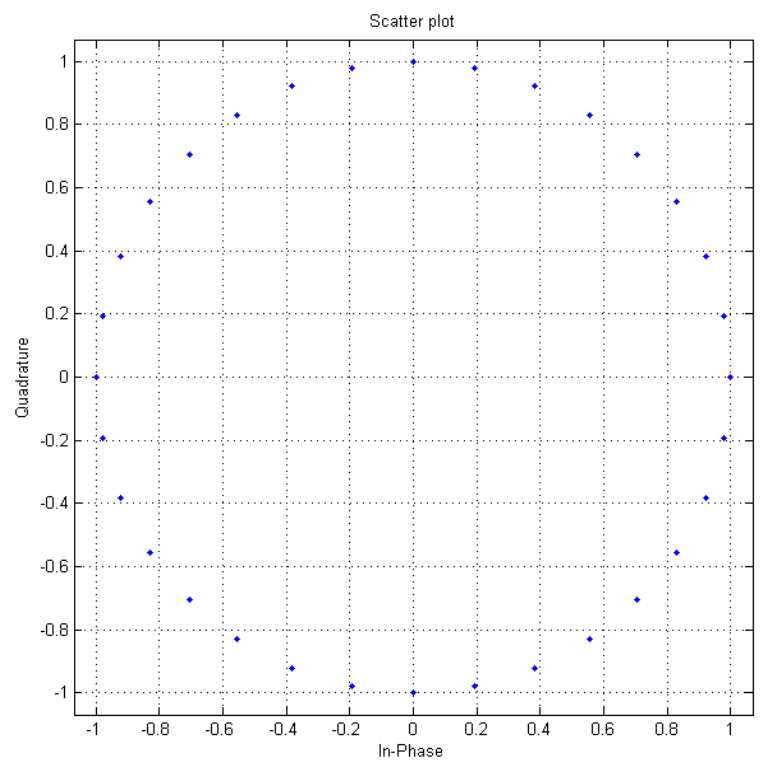

Figure 19: Constellation of 32PSK.

Figure 20. shows the first 100 input and output samples. The figure 21. shows the plot of input and output signal to visualize the error symbols. Figure 22 shows number of errors to respective Signal to noise ratio. Figure 23 show symbol error rate to corresponding Signal to noise ratios.
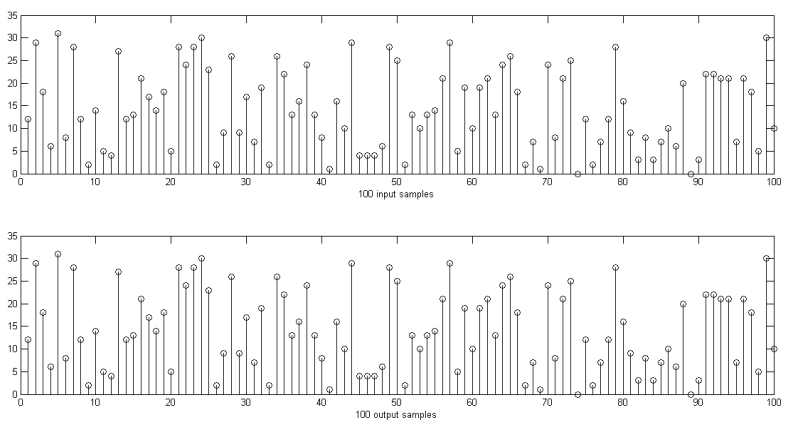

Figure 20: Input and Output samples

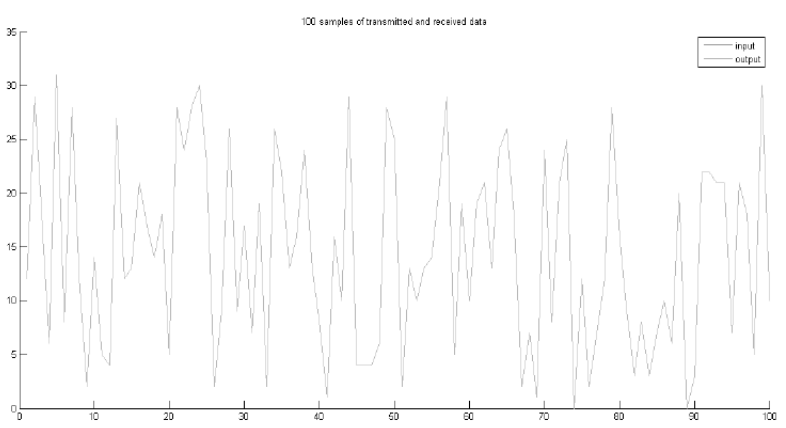

Figure 21: Plot of Input and Output signal to visualize errors.

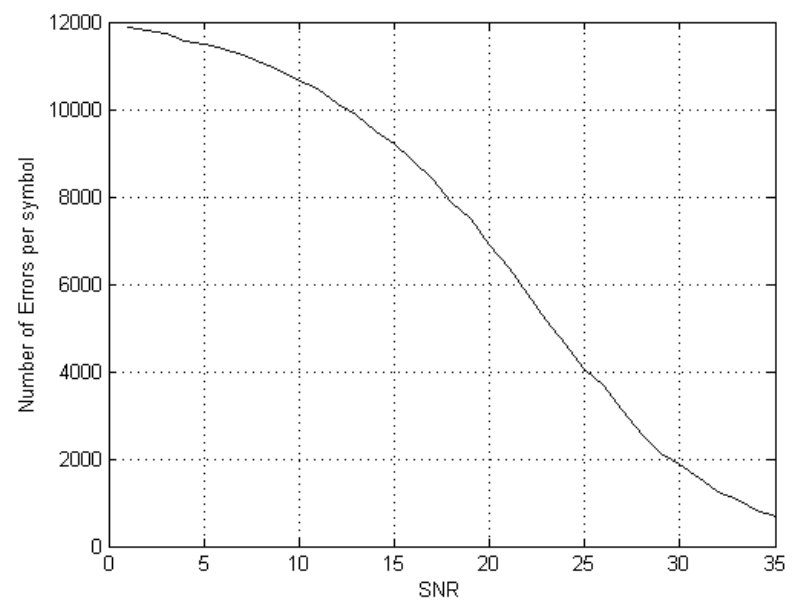

Figure 22: Number of error Vs Signal to noise ratio for 32psk-ofdm

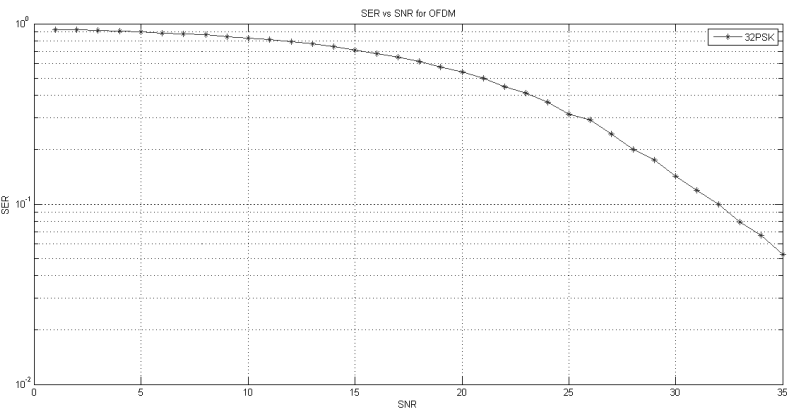

Figure 23: Symbol error rate Vs Signal to noise ratio for 32psk-ofdm. 
From figure 24. we can see that when we use high modulation level we have greater symbol error rate. 32PSK has greater symbol error rate but has higher data speed while using less channel bandwidth. We can reduce symbol error rate by increasing signal to noise ration. We can choose best modulation schemes to obtain optimum performance of the system based on needs, to obtain greater data rate we can choose higher modulation schemes and to have very less loss of data we can use lower modulation schemes.

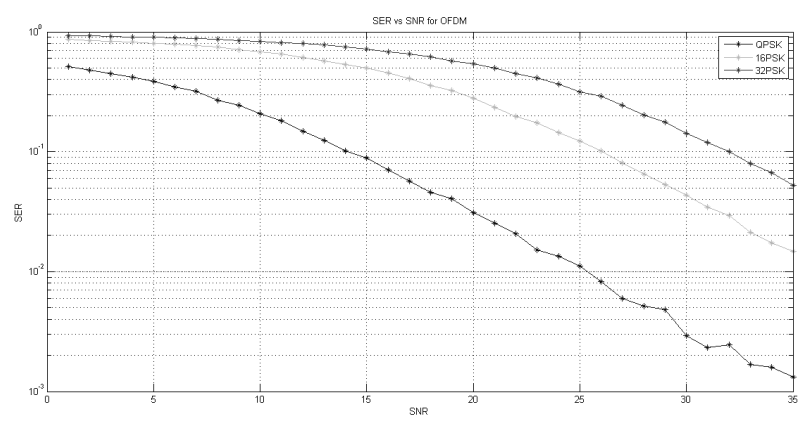

Figure 24: SER Vs SNR for PSK-OFDM

\section{CONCLUSIONS}

In this thesis we designed and investigated the performance of OFDM system at higher M-PSK(Phase Shift Keying) and M-QAM( Quadrature Amplitude Modulation). To compare different modulation techniques efficiencies it is important to calculate BER (Bit Error Rate) at different SNR(Signal to Noise Ratio). We observed from the results that we had more errors at receiver side when higher modulations are used because the symbols are located closely located in constellation diagram, we can reduce errors by increasing the SNR (Signal to Noise Ratio).From obtained results we can see that higher order M-QAM modulation gives better performance in AWGN than higher order M-PSK modulation.

In this paper we design and simulate OFDM (Orthogonal Frequency Division Multiplexing) to study the performance of the OFDM system at higher order modulation schemes. It is very important to evaluate performance of the communication system, to test the efficiency and quality of the service it can provide. We use Matlab program to design the functionality of OFDM, then BER(Bit Error Rate) is obtained to different SNR(Signal to Noise Ratio) values, for M-array PSK and M-array QAM modulations techniques. BER is widely used as performance measurement tool, it tells number of bits destroyed while the data is travelling from source to the destination, AWGN (Additive White Gaussian Noise) is used as transmission channel.

\section{REFERENCES}

[1] Digital Modulation in Communications Systems An Introduction. Hewlett Packard Company, P.O. Box 4026, Englewood, CO 80155-4026, 1997.

[2] Xiong and Fuqin. Digital Modulation Techniques. Artech House, Norwood, MA,USA, second edition, May 2006.

[3] Hiroshi Harada and Ramjee Prasad. Simulation and Software Radio for Mobile Communications. Artech House on Demand, 2002.

[4] Yong Soo Cho, Jaekwon Kim, Won Young Yang, and Chung-Gu Kang. MIMO-OFDM wireless communications with MATLAB. Wiley, 2010.

[5] L.J. Cimini, J. C-I Chuang, and N.R. Sollenberger. Advanced cellular internet service (acis). Communications Magazine, IEEE, 36(10):150\{159, 1998. ISSN 0163-6804. doi: $10.1109 / 35.722151$.

[6] H. Sari, G. Karam, and I. Jeanclaude. Transmission techniques for digital terrestrial tv broadcasting. Communications Magazine, IEEE, 33(2):100\{109,1995. ISSN 0163-6804. doi: 10.1109/35.350382.

[7] Simon Haykin. Communication Systems. John Wiley \& Sons, INC., third edition.

[8] Mike Fitton. Principles of digital modulation. URL http://www.berk.tc/combas/digital_mod.pdf.

[9] M. A. Mohamed, A. S. Samarah, and M. I. Fath Allah. Study of performance parameters e_ects on ofdm systems. International Journal of Computer Science Issues (IJCSI), 9(3):410, 2012. ISSN 16940784. URL http//proxy.lnu.se/login?url=http://search.ebscohost.com/lo gin. aspx ?direct $=$ true $\& d b=e d b \& A N=77651029 \&$ site $=$ edslive \&scope $=$ site.

[10] A. Pandharipande. Principles of ofdm. Potentials, IEEE, 21(2): $16\{19, \quad 2002$. ISSN 0278-6648. doi: 10.1109/45.997971.

[11] Yiyan $\mathrm{Wu}$ and W.Y. Zou. Orthogonal frequency division multiplexing: a multi-carrier modulation scheme. Consumer Electronics, IEEE Transactions on, 41(3):392\{399, 1995. ISSN 0098-3063. doi: $10.1109 / 30.468055$. 